\title{
Gefährdete Elternschaft und psychoanalytisch orientierte Entwicklungshilfe für Eltern-Kind-Beziehungen
}

\author{
Egon Garstick (Zürich)
}

\begin{abstract}
Zusammenfassung: Aus der Auseinandersetzung mit dem Thema Elternarbeit innerhalb der Kinder- und Jugendlichenpsychotherapie erwuchs dem Autor das Interesse an direkter Einflussnahme auf den frühkindlichen Bereich. In Zusammenarbeit mit einer sozialtherapeutisch tätigen Institution baute er eine flexible Kriseninterventionsstelle für psychisch in Not geratene Eltern auf. Anhand von zwei Fallbeispielen wird aufgezeigt, wie in komplexen SettingVerhältnissen mit Eltern, Säuglingen und Kleinkindern, psychoanalytische Entwicklungstheorien als Basis für die therapeutische Arbeit verwendet werden.
\end{abstract}

Schlüsselwörter: Dyadische und triadische Bedürfnisse von Babys und Eltern; Elternschaft; Wiederbelebung des Begehrens in der Elternbeziehung; Gewaltprävention; Auffangen von Projektionen und Ermöglichen des Entwicklungsraumes.

\section{$1 \quad$ Einleitung}

Ich werde mich auf die Darstellung meiner therapeutischen Arbeit mit werdenden oder gerade gewordenen Eltern konzentrieren, ohne dem Anspruch einer umfangreichen Auseinandersetzung mit theoretischen Fragestellungen gerecht zu werden. Vielmehr zeige ich dem Leser auf, wie ich im Laufe meiner psychoanalytischen Ausbildung und beruflichen Entwicklung immer mehr zu diesem einen Schwerpunkt meiner psychoanalytischen Arbeit gekommen bin.

Es waren Erfahrungen in der Arbeit mit gestrandeten, in ihrer Entwicklung blockierten, gestörten Kindern und ihren Eltern, die mir mehr und mehr von früh begonnenen Irritationen, Entgleisungen des Dialoges (vgl. Spitz 1976) und entscheidenden Veränderungen in der Qualität der Frau-Mann-Beziehung berichteten und mich begreifen liessen, wie wichtig psychoanalytische Reflexion und Hilfe in den frühen Phasen der Entwicklung sind. 
Einer meiner wichtigsten psychoanalytischen Lehrer, Pedro Grosz, hielt am psychoanalytischen Seminar Zürich (PSZ) schon ab 1981 Vorlesungen zum Thema «Elternarbeit in der psychoanalytischen Behandlung von Kindern». Er kritisierte die damals häufig anzutreffende naive Einstellung in weiten Kreisen der Kinderpsychotherapie, dass therapeutische Arbeit mit dem Kind allein bereits heilend wirke, ohne dass genauer darauf geachtet werden müsse, in welcherlei Loyalitätskonflikte die Kinder geraten und was für Abwehrmechanismen bei den Eltern aufkommen können, die schliesslich, wenn sie nicht konstruktiv aufgefangen werden, zum vorzeitigen Abbruch der Behandlung des Kindes führen können.

Er plädierte klar für ein Setting mit Einschluss der Eltern. So konnte ich bei ihm lernen, komplexe Übertragungsbeziehungen in den Interaktionen zu erkennen und damit zu arbeiten.

Pedro verstand die DreiergruppeVater-Mutter-Analytiker wie eine Gruppe, die sich zum Ziel gesetzt hat, die Geschichte des Kindes und dessen Abhängigkeit von den Biographien seiner Eltern besser zu verstehen.

Welche weiteren psychoanalytischen Autoren und Autorinnen beeindruckten mich und brachten mich weiter?

Als erstes fällt mir E. Abelin (1985) mit seiner Betonung der Funktion des Vaters ein. Ihm ging es um die psychische und kognitive Reifeentwicklung des Kindes, wofür seiner Meinung nach die gelingende Dreiecksbeziehung zwischen Eltern und Kleinkind benötigt wird.

Dann denke ich an J. Stork (1986) mit seinen Arbeiten über den «Vater als Befreier und Störenfried» und an das Werk von F. Dolto (1987), die u.a. immer wieder grossen Wert auf die Qualität der Elternbeziehung legte. Sie interessierte sich z. B. dafür, ob nicht ein Elternteil das Kind unbewusst zu sehr als seinen Besitz begreift und keine libidinösen Besetzungen der Frau-Mann-Beziehung mehr vornehmen kann.

Ganz wichtige Anstösse für die psychoanalytische Arbeit mit Eltern und Babys gaben mir auch die Kinderanalytiker Bianca Gordon und Sjef Teuns, die ab Ende der 70er Jahre periodisch aus London und Amsterdam zur Förderung der Ausbildungsqualität im Bereich Kinderpsychoanalyse ans PSZ eingeladen wurden.

Sie lehrten uns, wie offen die meisten Mütter nach der Geburt für die Bearbeitung wichtiger Lebensthemen sind und wie wichtig deshalb fokussierte psychoanalytische Arbeit sei, um den Frauen zu helfen, ihre eigenen inneren 0bjektbeziehungen zu verstehen und die sich daraus ableitenden möglichen Störungen für die aktuelle Beziehungsaufnahme zum Baby zu durchschauen. Beeindruckt 
von den Erkenntnissen um die Bedeutung der frühkindlichen Entwicklung, begann ich damit, sie auch in sozialpädagogische und sozialarbeiterische Arbeitsfelder hineinzutragen.

Die Stiftung «Mütterhilfe Zürich» interessierte sich ganz besonders für diese psychoanalytischen Entwicklungstheorien, wagte Schlussfolgerungen zu ziehen für den eigenen Erfahrungsbereich und setzte sich ernsthaft mit der Frage ihrer Anwendbarkeit in ihren Arbeitsfeldern auseinander. Der primäre Auftrag der Mütterhilfe besteht darin, möglichst viele Mütter in sozialen und materiellen Notsituationen erst einmal aufzufangen und sie in ihren Versuchen, für sich und ihre Kinder eine menschengerechte Existenz aufzubauen, zu unterstützen. Nun sollte diese wichtige sozialarbeiterische Hilfe durch sozialtherapeutische Interventionen erweitert werden. Unser Augenmerk richtete sich auf die Beziehung zwischen Mutter und Kind. Wir fühlten uns verantwortlich, die in Not geratenen Mütter, mehrheitlich waren es Alleinerziehende, darin zu unterstützen, ihrem Baby eine gute Entwicklung zu ermöglichen.

Hebammen, Geburtsabteilungen von Kliniken, Kinderärzte und diverse Beratungsstellen wurden auf uns aufmerksam und nahmen unsere neue Art von Sozialtherapie in Anspruch:

Zwei Psychotherapeutinnen besuchen Mütter und Kinder zu Hause, wenn sich das z. B. bei einer postnatalen Depression oder einer anderen psychiatrischen Diagnose der Mutter als sinnvoll erweist.

Später realisierten wir, wie wichtig es ist, auch die Väter zu erreichen, sei es in unklaren, voneinander getrennt lebenden Paaren, aber auch in sogenannten vollständigen Familien. Ich bekam den Auftrag, ein Behandlungskonzept zu entwickeln, um den frischgebackenen Elternpaaren oder den auch schon durch die Schwangerschaft herausgeforderten Paaren zu helfen.

\section{$2 \quad$ Kurzer Abriss eines Referenzsystems zur Elternschaftstherapie}

Es geht mir um die Anwendung entwicklungstheoretischen Wissens. Das Baby braucht einerseits die Empathie seiner Eltern, aber es braucht andererseits - auch schon in seiner frühen Entwicklung - das Erleben von Eltern als sich begehrende Subjekte (Paarbeziehung, Interessen über das Baby hinaus). Wenn die Eltern sich nicht auch als Mann und Frau wieder finden, ist ein triadisches Entwicklungsklima für das Kind gefährdet (vgl. Dammasch 2008). Am Anfang meiner Erfahrungen in diesem Bereich, stiess ich in psychoanalytischen Kreisen auf die besorgte Frage, wie ich in diesen komplexen Mehrpersonen-Settings meine psychoanalytische Identität wahren könne. Einerseits bin ich mit dem Wissen 
um die Bedeutung der unmittelbaren Bedürfnisse des Säuglings resp. Kleinkindes identifiziert und andererseits auch mit seinem Entwicklungsanspruch nach triadischen Beziehungen und Entwicklungsräumen. Dazu gehört die Bedeutung des wiederentstehenden Begehrens zwischen Mutter und Vater.

Nur wenige AutorInnen beschreiben klar und deutlich die Bedeutung der Qualität von sinnlichen, sexuellen Erfahrungen in der Elternbeziehung von Säuglingen, als ob trotz Aufklärung die Sexualität und ihr Abflauen in Elternbeziehungen tabuisiert werden müsste. Ich mache die Erfahrung, dass Frauen und Männer dankbar dafür sind, wenn sie in ihrem Beziehungspsychotherapeuten (Begriff in Anlehnung an Bauriedl (1994)) jemanden gefunden haben, der sich neben der Feinfühligkeit gegenüber dem Baby auch für das «Kind» der libidinösen Mann-Frau-Beziehung interessiert und dafür sorgt, dass der Diskurs darüber lebendig bleibt. Die Entwicklung des Kindes ist auch vom Wohlbefinden seiner Eltern abhängig. Es kommt sehr darauf an, in welcher Weise sie die Anwesenheit des Babys und die Veränderung ihrer Paarbeziehung erleben. MancheVäter erleben das Kind schon früh als Rivalen. Ein Vater drückte es treffend aus: «Da habe ich mir etwas Schönes eingebrockt. Ich habe gezeugt und verloren! Mein Sohn hat nun so intensiv die Mama, und ich habe eine schöne Frau verloren!» Der Mann hatte u. a. enorme Mühe mit den grossen Brüsten seiner stillenden Frau und mit ihrem durch Schwangerschaft und Geburt veränderten Körper. Er war eine Zeit lang regelrecht depressiv und verhielt sich stark unterkühlt gegenüber Baby und Frau.

Bei starken, länger andauernden Abkühlungen und Entfremdungen in der Frau-Mann-Beziehung besteht die Gefahr eines grossen Verlustes für das Kind.

Für den argentinischen Psychoanalytiker Ricardo Rodulfo (1996) ist die komplexe Entwicklung der Körperseele davon abhängig, ob es gelingt, «sich als Frucht einer vom Begehren bestimmten Begegnung einer gewissen Intensität fassen zu können. Wie wir wissen, ist es für ein Subjekt entscheidend, dass es auf einer im strengsten Sinn unbewussten Ebene etwas gibt von der Art: Dein Ursprung war ein Augenblick der Lust, eine Zeit erotischer Begegnung, die von einem Paar erlebte Lust» (1996: 94).

Ein Kind kann sich nicht als gewünschtes Subjekt erleben, wenn die Entgleisungen im körperlich-seelischen Dialog zwischen Mann und Frau in der Elternbeziehung nicht bearbeitet werden. Hier versuche ich, psychoanalytisch anzusetzen.

Christiane Olivier (1988) beschreibt eindrücklich die Gefahr der unaufgearbeiteten Enttäuschungen in der Mann-Frau-Beziehung während der Schwangerschaft und nach der Geburt mit allen möglichen Folgen pathologi- 
scher Mutter-Sohn-Beziehungen, deren Qualitäten von Generation zu Generation unbewusst weitergegeben werden können.

Wenn die Paarbeziehung nicht wieder befriedigend aufgenommen werden kann, beeinträchtigt dies die Besetzung der generativen Rolle bei beiden Elternteilen (King 2004).

Nach diesem grob skizzierten Überblick über wichtige theoretische Bausteine, möchte ich zur näheren klinischen Diskussion kommen.

Für meine therapeutische Arbeit in diesem komplexen Feld war sicher auch dieWeiterbildung in körperorientierter Psychotherapie bei George Downing (1996) unterstützend. Hier lernte ich ein aktiveres Explorieren von Körperempfindungen. Das ist deshalb von Bedeutung, weil für mich der Aufbau eines differenzierteren Selbstempfindens im Beziehungsdreieck Mutter - Vater - Baby wichtig ist.

Körperselbst-Wahrnehmung ist eine Bedingung für elterliche Feinfühligkeit und die Entwicklung der Mentalisierungsfähigkeit beim Baby (vgl. Fonagy 2004). Um gravierende Beeinträchtigungen zu verhindern, ist bei Depressionen eines Elternteils, besonders rasche Hilfe für die frühe Beziehungs-Entwicklung indiziert.

Die meisten mir bekannten Beratungsstellen gehen in ihren Indikationen zu Eltern-Säuglingspsychotherapien bei Schrei- und Schlafproblemen der Babys davon aus, dass nach dem Wegfall von somatischen Befunden eine angeborene oder während der Schwangerschaft erworbene, besondere Empfindlichkeit des Babys, gekoppelt mit einer unbewussten Konfliktlage der Mutter, angenommen werden kann (Knott 2003). Ich erweitere diese Indikationsbeschreibung durch den Zusatz, dass wir auch eine unbewusste Konfliktlage in der Mann-Frau-Beziehung in Betracht ziehen müssen, die von den verschiedensten äusseren oder verinnerlichten Objektbeziehungen aus den jeweiligen Herkunftsfamilien der Eltern herrühren kann.

Für diese vielfältigen Problemfelder muss ich mich als Psychoanalytiker interessieren, wenn ich schreienden Babys, verzweifelten Müttern und «abgelöschten»Vätern begegne.

Ich nenne dies «psychoanalytische Elternschaftsarbeit». Das heisst für mich, dass ich mit meinem Wissen um die Bedeutung innerer Beziehungsmodelle, Projektionen und Übertragungen an die Dyaden und Triaden herangehe, um zu schauen, wo und wie die frühkindliche Entwicklung des werdenden Subjektes (Fötus, Baby, Kleinstkind) gestört wird.

Es geht auch darum, mögliche Gewalt in frühen Beziehungen aufzufangen, die Eltern zu unterstützen, ihre Affektkontrolle wieder aufzubauen, die oft durch 
länger andauerndes Schreien des Babys aus den Fugen geraten ist (vgl. Garstick 2001).

Wie schon erwähnt, verändern sich bei Mann und Frau die libidinösen Besetzungen. Damit können sie mit ihren Wünschen, Anlehnungsbedürfnissen und libidinösen Erwartungshaltungen sowie mit unbewussten Identifizierungen in Schwierigkeiten geraten.

Ich durfte die Erfahrung machen, dass auch die Männer in diesen vor- und nachgeburtlichen Lebensphasen ihrer Frauen gegenüber Introspektion und dem Sich-Erinnern offener sind.

Als Psychoanalytiker stelle ich mir die Aufgabe, entscheidende Entwicklungsabschnitte in der Geschichte eines Elternteils aufzugreifen und mit den Eltern darüber zu reflektieren, wie diese Erlebnisse mit Fehlbesetzungen oder Besetzungsunmöglichkeit in der realen Situation mit dem Baby zusammenhängen könnten.

\section{$3 \quad$ Fallbeispiel Maria und Paul}

Beide waren zum Zeitpunkt der Therapie etwa 35 Jahre alt. Sie suchten die Schwangerschaftskonfliktberatung meiner sozialarbeiterischen Kollegin in der Beratungsstelle Mütterhilfe auf, weil sie sich unsicher waren, ob sie ein drittes Kind wollten. Die Frau war ungeplant schwanger geworden und das Paar dachte über einen Abbruch nach.

Aus dem 2. Beratungsgespräch, in dem die Frau mehr und mehr realisierte, dass sie das Kind austragen wollte, rannte der Mann davon, weil er sich einer weiblichen Übermacht ausgeliefert fühlte. Bevor er aber definitiv den Raum verliess, konnte meine Kollegin ihm noch mein Kärtchen mit der dringenden Empfehlung geben, sich bei mir zu melden, was er auch tat.

Eine Zwischenbemerkung: In dieser Arbeit mit Eltern und Säuglingen während der Schwangerschaft und der Zeit nach der Geburt ist es besonders wichtig, für das interdisziplinäre Netz von Kinderärzten, Mütterberaterinnen und Sozialarbeiterinnen rasch und verbindlich erreichbar zu sein.

Zurück zur Fallgeschichte: Wie wirkte Paul in der ersten Begegnung auf mich? Ich sah einen verzweifelten, verwirrten, erröteten, übernächtigt und erschlagen wirkenden Mann vor mir. Andererseits nahm ich auch einen grossen, offen wirkenden Mann wahr, der rasch das Gespräch suchte, aber auch unter einem körperlich spürbaren Druck zu stehen schien.

Ich bemerkte diesen Druck in mir selbst und versuchte, zwei Ebenen zu trennen. Die Wahrnehmung seiner Gehetztheit, die klar seinem Stress zuzuordnen 
war, einerseits und andererseits meine Bemühung darum, selbst entspannt zu bleiben fürs analytische Zuhören seiner Geschichte.

Ich vernahm von seiner sozialpolitischen Tätigkeit und seinen vielfältigen kommunikativen Aufgaben und erlebte, wie er sich rasch und anschaulich mitteilen konnte, dabei realisierte ich in seiner Schilderung auch eine gute Portion Ironisierens. Er zeigte überdies starke Anlehnungsbedürfnisse, wollte in seiner Not verstanden werden und konnte gar nicht mit dem Erzählen aufhören. Er musste Dampf ablassen und schien sich im Laufe des Gespräches ein Stück weit zu beruhigen, was für mich selber körperlich spürbar wurde.

Er begann mit folgender Eröffnung: «Ich bin doch schon glücklicher Vater von zwei tollen Jungs, 3 und 5 Jahre alt. Ich habe eine wunderbare Frau, mit der ich nun langsam wieder mehr Zweisamkeit erleben wollte und nun soll noch ein Wuschel dazu kommen. Der bringt doch alles aus dem Konzept! Ich verstehe diese Haltung meiner Frau einfach nicht. Kann sie sich nicht mehr daran erinnern, wie anstrengend die Aufzucht unseres zweiten Sohnes war, wie alles ausser Rand und Band geriet: Schlafen nur noch 2-3 Stunden pro Nacht. Ich stand kurz vor einer Einweisung in die Klinik».

Der Mann schilderte mir eine grosse Angst vor einer erneuten totalen Überforderung. Ich erfuhr ausserdem, dass er damals in der frühen Entwicklungsphase des zweiten Sohnes auch noch in eine berufliche Krise geraten war und sich ernsthafte Sorgen um die materielle Existenz gemacht hatte.Bei genauerem Nachfragen stellte sich heraus, dass die junge Familie dank ihrer solidarischen Grosseltern mütterlicherseits nie wirklich vom Absturz ins Elend bedroht gewesen war. Dem Vater war allerdings die materielle Autonomie in der Heimat seiner Frau sehr wichtig. Er habe mit seiner Frau, in einer grossen Stadt seines Herkunftslandes, herrliche, verliebte und anregende Jahre verbracht. Daraufhin sei der Kinderwunsch bei seiner geliebten Frau stark aufgeblüht. Lange Zeit sei es ihm schwer gefallen, diesen Wunsch zu teilen und seine ablehnende Haltung habe zu einer Krise in der Paarbeziehung geführt. «Ich aber wollte Maria unbedingt, dann eben auch mit Kindern. Mit dem ersten Bub ging es schon noch, aber es war bereits in der Schwangerschaft nicht mehr so gut. Maria ging mir ein Stück weit als Frau verloren. Der Bauch und die grossen Brüste, ich habe Mühe damit!»

Er zeigte in diesem Moment beim Erzählen Ekelgefühle und Hilflosigkeit.

Ich hörte, wie es zur aktuellen Schwangerschaft gekommen sei: «Vor einem Jahr musste die Spirale bei meiner Frau entfernt werden. Ich wusste vom Risiko, ungeschützt mit ihr zu schlafen. Ich wusste von ihrem tief verankerten Wunsch nach einer grossen Familie. Sie wollte ja ursprünglich mal vier Kinder. Ach, ich hätte 
mich doch sterilisieren lassen sollen! Nun muss ich wohl die Suppe auslöffeln. Diese Macht meiner Frau hat mich einfach umgehauen. Ich kann sie auch heute noch nicht wieder richtig anschauen, wie sie mich vor vollendete Tatsachen setzt und mich allein lässt mit meinen Ängsten, z. B. mit der Angst um unsere Beziehung, dass für die gar kein Platz mehr bleibt.»

In dem Moment wirkte er wirklich wie ein total eingefallener, erschlagener Mann, der sich ausgeliefert fühlte. Ich dachte, neben den real berechtigten Sorgen, an dahinter liegende unverarbeitete Überwältigungserfahrungen und mir gingen folgende Gedanken durch den Kopf:

Er hat mir seine Sorgen um den Verlust seiner Frau geschildert und von der Angst vor dem Abgleiten in ein spiessiges, kleinbürgerliches Leben erzählt. Ich habe von ihm gehört, wie er auf einem Bauernhof in einer sehr provinziellen Gegend gross geworden ist. Die Distanzierung von seinem Vater und dem konservativen, zum Teil sprachlosen Umgangsstil zu Hause ist ihm sehr wichtig. Er sei richtig aufgelebt in der Grossstadt und habe sein Leben mit seiner Maria dort gefunden.

Ich wollte nun diese tiefe Verzweiflung, die irgendwie über den Ärger auf seine reale Frau hinaus ging, besser verstehen. Er hatte mir ja erzählt, dass er nach dem entscheidenden Gespräch bei unserer Sozialarbeiterin wie betäubt durch die Strassen geirrt war. Offenbar fühlte er sich überwältigt. Deshalb versuchte ich, einen erweiterten Wahrnehmungsraum zu entwickeln. Ich kam nochmals auf die geschilderte Panikstimmung zu sprechen, atmete selber bewusst tiefer und ruhiger und versuchte, einen Perspektivenwechsel vorzunehmen.

Ich fragte nach, ob er diese starke Verzweiflung, diese heftigen Affekte, eventuell anderswoher auch kenne: «Woran erinnert Sie vielleicht dieses so starke Überwältigtwerden? Da «mutet` ihnen eine Frau etwas zu. Kennen Sie das?!»

Paul konnte meinen Versuch, für ruhigeres Atmen zu sorgen, annehmen. Er ging in sich und es fiel ihm etwas ein. Er war sichtbar gerührt, seine Augen wurden feucht, in mir tauchte Traurigkeit auf.

Da er verlegen wirkte, sprach ich kurz mit ihm über seine Schamtoleranz. Ich hörte, solche Gefühle, geschweige denn Tränen, könnte er normalerweise schlecht in Anwesenheit eines Anderen zulassen. Aber nun wäre es schon gut hier und er konnte Folgendes sagen: «Da haben Sie etwas getroffen. Es sind die gleichen verzweifelten Gefühle wie damals, als ich von meinen Eltern zu Hause wieder in die grosse Stadt zurückfuhr und realisieren musste, dass ich eine sterbende Mutter zurücklasse und sie mir verloren geht. Meine Mutter hatte Krebs und musste 60 -jährig schon sterben. Meine Frau war mit unserem ersten Bub schwanger, als dann meine Mutter starb.» 
Wir schwiegen beide ergriffen eine Zeit lang, während er die Tränen zulassen konnte. Er war ruhig geworden, körperlich ruhiger, auch für mich wahrnehmbar.

Ich sagte dann: «Beide Male wurden Sie überwältigt. Ihre Mutter hat Ihnen ihr Sterben «zugemutet und nun mutet Ihnen Ihre Frau die Annahme eines weiteren Kindes zu. Die Entscheidung Ihrer Frau fürs Kind wird von Ihnen wie eine wichtige Verlusterfahrung erlebt!»

Er ging gelöst, nach diesem ersten Kontakt, bedankte sich und konnte sich wieder etwas Nähe zu seiner Familie vorstellen. Es standen Weihnachtsfeiertage bevor und er dachte darüber nach, wie er sich Zeit und Raum verschaffen wollte, um seiner Frau davon zu erzählen, was ihm in dieser ersten Stunde passiert war.

\section{Reflexion der ersten Stunde}

Das Deutlichwerden der Erfahrung des Ineinandergreifens, des sich Vermischens von Ohnmacht gegenüber dem Tod, des Verlustes der Mutter, mit der Zuwendung zum Leben mit der schwangeren Frau, war beeindruckend. Die Mutter hatte ihn, im Gegensatz zum Vater, offenbar auch gut in seinem Distanzierungswunsch vom bäuerlichen Leben im Heimatdorf, verstanden. Nun hatte er die ihn in seinen Autonomiewünschen verstehende Mutter zu Gunsten des neuen Lebens verlassen.

Daneben faszinierte mich ein anderer Aspekt: Die von ihm so stark begehrte Frau Maria wird für den um Autonomie ringenden Mann mit ihrem Kinderwunsch offenbar «gefährlich». Sie mutet ihm die Übernahme von Generativät zu. Seine generative Rolle als Vater zu übernehmen, heisst auch für den Mann, neben möglichem narzisstischem Gewinn, die Begrenztheit der Existenz und das Teilen der libidinösen Aufmerksamkeit im triadischen Feld zu akzeptieren. Ich war fasziniert von dieser Überdeckung, diesem Ineinanderfliessen von den beiden Themen Tod und Leben.

Parallel zu dieserWahrnehmung seiner existentiell anmutendenVerzweiflung wurden mir auch die negativen Gegenübertragungsgefühle klarer.

Was hatte er Nervendes ausgelöst? Machte mich sein abwertendes Beschreiben derVeränderung des weiblichen Körpers durch die Schwangerschaft ärgerlich? War ich schon ein Stück weit mit seiner Frau, die mir ja als sehr schön beschrieben worden war, identifiziert? Was störte ihn an einem grossen Busen, an grossen Brustwarzen? Ging es um verdrängte homosexuelle Seiten? Verdrängte homosexuelle Seiten, die zu einer Entwertung der heterosexuellen Erotik zwischen Mann und Frau führen? 
Er störte offensichtlich meine eigene generative Haltung und meine Freude an der Erotik eines schwangeren Körpers. Nach der Klärung dieser Gegenübertra gungsgefühle, konnte ich mich neugierig auf den interessanten Einblick in das Erleben eines Mannes einlassen, der sich mit der generativen Rolle schwer tut.

Beeindruckt war ich hingegen von seiner Offenheit, mit der er sagen konnte: «Ich will auch wieder mehr wahrgenommen werden!»

Meine Erfahrung mit Paaren, die Eltern werden, mit Männern, die Väter werden, ist häufig die, dass die Veränderung des Begehrens nicht kommuniziert, sondern nur diffus erlebt und meistens missmutig und nicht mehr konstruktiv mitgeteilt wird.

Oft erleben Männer die Veränderung des weiblichen Körpers als Verlust. Das Kind raubt ihnen die Geliebte. Diese Wahrnehmungen brauchen eine fokussierte Bearbeitung in einem Holding, damit die erlebten Verluste gemeinsam betrauert werden und Besetzungen für den veränderten Körper der Frau und die neue Lebenssituation frei werden können.

\section{Zurück zur Fallbeschreibung}

Über die Weihnachtsferien gab es eine Pause in der weiteren Arbeit und Paul kam erst zwei Wochen später zum nächsten Termin. Er berichtete von seiner Unzufriedenheit über den Rückgang des Sexuallebens in ihrer Beziehung. Wir besprachen seine Irritationen, merkten aber auch, dass er mit seiner Ablehnung des Wunsches nach einem weiteren Kind, zwischen sich und seiner Frau eine Entfremdung eingeleitet hatte. Wir besprachen, wie er seine Wünsche nach Nähe wieder in die Beziehung einbringen könnte und dank seinem Humor liess sich feststellen, dass ein Gejammer über sexuellen Notstand die Frau nicht unbedingt animieren würde. Nach zwei weiteren Stunden berichtete er von einer Wiederaufnahme der sexuellen Beziehung.

DerVater erzählte mir beeindruckt und gerührt über seinen ältesten, 5-jährigen Sohn, der wohl etwas vom Konflikt der Eltern ahnte und seinen Papa fragte:

«Gibst Du der Mama wieder mal einen Samen, wir wollen noch gern ein Geschwisterchen!»

Im weiteren Verlauf kam es zur Erweiterung des Settings. Die Frau wollte mit ihm zu mir kommen, was der Mann begrüsste. Offenbar fühlte er sich durch unsere Arbeit zu zweit genügend verstanden und war für die triadische Begegnung bereit. 


\section{Das Paar bei mir}

Die Dreierbeziehung ermöglichte beiden, sich besser mitzuteilen als zu Hause. Maria realisierte, dass sie fast aufgehört hatte, ihrem Mann von ihrem Erleben oder ihren Wünschen zu erzählen. Damit fühlte sie sich einsam in ihrer Beziehung und wagte nicht mehr, ihre Anlehnungsbedürfnisse zu zeigen. Daneben, und das war ein ganz wichtiger Gedanke, gefiel ihr aber sein adoleszent wirkender Teil, der rebellische, rhetorisch gekonnt ironisierende Mann.

Reflexion meiner Gegenübertragungsgefühle und der sichtbar gewordenen Dynamik

Maria kam mir wunderschön vor. Ich dachte: «Du Kostverächter verdienst sie gar nicht! Wie kann er nur so blind sein? Mit dieser Frau könnte er es doch gut haben.»

Da wurde ich ödipal herausgefordert. Daneben liessen mich väterliche Gefühle ihr gegenüber denken, er solle sich doch mal vernünftig um sie kümmern. Neben all der ödipalen väterlichen Verehrung der bildhübschen Tochter tauchte aber auch ein kritischer Gedanke ihr gegenüber auf.

Ich erwähnte ja schon, dass sie diesen adoleszenten Teil in ihrem Mann auch belebend fand. Ich meinte, entdeckt zu haben, wie sie sich an entscheidenden Stellen zu sehr an die von ihm in die Beziehung hinein gebrachte Ironie anpasste und auf diese Weise wohl vieles von ihren wichtigen Bedürfnissen auf der Strecke blieb.

Mit Hilfe unserer Arbeit realisierte sie dies und nahm sich vor, ihre Wünsche nach Beachtung und Fürsorglichkeit ihm gegenüber zu formulieren. Sie wäre gefährdet gewesen, den Dialog mit dem Kind im Bauch gar nicht genussvoll führen zu können, weil sie bei den Schwierigkeiten ihres Mannes mit seiner erneuten Vaterschaft Mühe hatte, alles weitere vertrauensvoll auf sich zukommen zu lassen. Hier zeigt uns Maria, wie wichtig eine rechtzeitige psychotherapeutische Arbeit sein kann. Die Intervention ermöglichte es, die libidinöse Besetzung des heranwachsenden Kindes zu verstärken und Wünsche und Begehren in verschiedenen Bezügen wieder aufleben zu lassen.

Weiterer Verlauf der Fallgeschichte

Paul wurde eine Woche vor dem errechneten Geburtstermin von seiner Frau zu einem Treffen mit Freunden geschickt. Er sollte sich nochmals austoben und reiste hierfür eine Tagesreise entfernt in eine ausländische Stadt. 
Ich sah das Paar mit Tochter Nina erst wieder vier Wochen nach der Geburt. In einem vorangehenden Telefongespräch hatte ich bereits erfahren, dass die Familie weiblichen Zuwachs bekommen hatte. Ich dachte: «Uff, noch mehr Frauen, aber Schwierigkeiten gab es ja vor allem mit dem zweiten Jungen in dessen Säuglingszeit!»

Beide Eltern strahlten erst einmal, als ich sie begrüsste und machten es sich im Praxisraum gemütlich. Zu Beginn der Sitzung befand sich Nina in den Armen der Mutter. Diese nutzte bald den Raum bei mir, um ihre Wut auszudrücken. Sie hatte anfänglich Schuldgefühle wegen ihrer Aggression, doch ihr Mann fand, sie solle hier ruhig alles raus lassen. Es machte ihr sichtbar Angst, deutlich zu sagen: «Ich hätte Dich schlagen können!»

Sie habe auch noch in den letzten Nächten ganz wütende Träume gehabt, in denen sie ihren Mann heftig geschlagen habe. Als sie dann traurig wurde, weil sie von ihrer Erfahrung des Sich-Verlassen-fühlens berichtete, gab sie dem Vater die kleine Tochter.

Er legte sie liebevoll auf seine Brust und verhielt sich auch im weiteren Verlauf positiv unterstützend. Seine Frau bekam den Raum, um ihre Verletztheit darzustellen. Sie berichtete, dass ihre Enttäuschung da schon angefangen hätte, als er in sein time-out (Kollegentreffen im Ausland) gefahren sei, obwohl sie den besorgniserregenden Befund der Plazenta-Insuffizienz bekommen hatte und in der Folge viel habe liegen müssen. Sie sei hin- und hergerissen gewesen. Hätte sie es ihm zumuten dürfen, bei ihr zu bleiben? Oder wäre er dann doch so enttäuscht gewesen, dass er nach der Geburt ständig genervt gewesen wäre und ihr indirekt immerVorwürfe gemacht hätte? Sie habe ihn fahren lassen und sich in Obhut einer Lieblingstante und ihrer Mutter begeben. Daraufhin berichtete Maria von einem sehr langen, anstrengenden Geburtsverlauf, in dem sie ihren Mann als zu müde und wenig hilfreich erlebt habe. Er habe sich nämlich in dieser Männerclique bei dem grossen Fest ziemlich «zugedröhnt» mit Alkohol und Drogen und sei total verkatert zurückgekehrt.

Sie habe dann in dem schwierigen Geburtsverlauf - Gott sei Dank - eine gute Hebamme gehabt und im Gespräch mit der Frau sei es ihr klar geworden, wie schwer es ihr gefallen sei, das Kind loszulassen. Maria sprach offen über ihre radikalen Gedanken, die ihr selber Angst gemacht hatten. Ihre Phantasie bei der Geburt war, dass sie das Kind nicht von sich lösen könne, weil sie es diesem Vater, der es gar nicht gewollt hatte und ihr gegenüber so wenig fürsorglich war, nicht anvertrauen könne. Daher sei sie auch heute noch voller Wut. 
Sie fragte uns beide aufgewühlt, ob sie mit dieser Wut verstanden werden könne und ob sie jetzt gleich wieder verzeihen müsse? Paul zeigte sich gerührt und nahm ihre Wut an. Maria war etwas versöhnt. Ich fand, dass der Vater mit seiner Haltung im Umgang mit dem Baby eine Kompetenz zeigte, die uns erahnen liess, dass er in Zukunft auch seine Frau besser würde halten können.

Zum Schluss einigten wir uns darauf, dass der Vater für die nächste Stunde allein zur Sitzung komme, weil er seine «Aussetzer», so nannte er selbst sein Verhalten bei der Geburt, noch genauer verstehen wolle. Es wurde aber auch ein weiterer gemeinsamer Termin von Maria und Paul bei mir vereinbart. Ich hatte das Gefühl, ihm auch noch etwas mitgeben zu müssen, nachdem die Mutter viel Trost und Verständnis gefunden hatte.

Ich sagte zu ihm: «Sie sind ein reicher Mann. Sie haben da eine so herzige kleine Tochter so schön bei sich, Sie haben zwei Söhne und eine schöne Frau, die sich Ihnen mutig und ehrlich mitteilt.» Damit hoffte ich, seine noch fragile väterliche Identität etwas zu unterstützen.

Eine Woche später kam der Vater allein: die Kritik seiner Frau habe ihm schwer zu schaffen gemacht. Er sei bestürzt und habe Angst bekommen, seine Frau zu verlieren. Meine Worte über seinen Reichtum hätten ihn schon erreicht.

Er meinte: «Maria und ich haben bereits nach der Geburt des anstrengenden zweiten Kindes so viel Zeit verloren für den Wiederaufbau unserer Liebesbeziehung. Wie schaffen wir das jetzt nach dem dritten Kind?» Ich erklärte, dass dies eher zustande kommen könne, wenn er von seiner Frau auch als fürsorglicher Vater und Partner erlebbar werde. Er äusserte die Angst, dass sie gar nicht mehr an der Entwicklung der Paarbeziehung interessiert sei. Ich konnte ihn aber daran erinnern, wie sie uns beiden beim letzten Treffen davon erzählte, wie sie sich auf den ersten gemeinsamen Ausgang zum Geburtstagsfest eines Freundes gefreut habe. Sie habe sich gerne stolz mit ihm gezeigt. Dann beschäftigte sich Paul mit der Frage, was er für sich tun könne, damit er seine Frau nicht erneut so sehr verletze, wie in der Zeit um die Geburt des dritten Kindes. Paul wörtlich: «Was kann ich tun, damit Maria wieder Vertrauen zu mir fasst?» Selbstkritisch fragte er sich, was dies alles mit seiner Herkunftsfamilie zu tun haben könnte. Meine Aussage über seinen heutigen Reichtum habe ihn auf etwas gebracht. Sein Vater suchte ständig Bestätigung und dabei hätte er, sein Sohn, diese doch von ihm so dringend gebraucht und nie erhalten.

Paul: «Suche ich da noch eine Auseinandersetzung mit dem Vater? Bin ich deshalb immer mal wieder so schwierig für meine Frau?» 
Er wollte an diesen Themen intensiver arbeiten. Der junge Vater realisierte die Macht der inneren unbewussten Konflikte mit den Objekten. Seine nicht verarbeiteten ödipalen Konflikte mit dem Vater und die mangelnde Besetzung durch ihn hatten sich störend in die Entwicklung von Generativität eingeschlichen. Für ihn war Elternsein stark mit Selbstverlust in der Dyade mit seiner Frau verbunden. Später, nachdem sich die familiäre Situation beruhigt hatte, gelang es mir den Vater in eine weiterführende psychoanalytische Psychotherapie bei einem Kollegen zu überweisen.

Worum ging es mir als Psychoanalytiker bei dieser Arbeit mit Maria, Paul und ihren Kindern?

Ich wollte einer jungen Familie mit einem dekompensierenden Vater ein Holding (Winnicott 1974) bieten und damit unter anderem die zu erwartende Gewalt oder Entfremdung in der Familie verhindern. Solche präventiven Massnahmen sind in einem interdisziplinären System viel besser und rascher möglich. Es entspricht Winnicotts Überzeugung, dass eine sich in Verzweiflung befindende Mutter mit ihrem Säugling nicht eine Woche warten gelassen werden darf. Wir übertragen diesen Anspruch auch auf die Arbeit mit Vätern.

Der beschriebene Vater hätte sich selbst gefährden und seiner Familie als Vater verloren gehen können. Das noch ungeborene Kind war in Gefahr, unter enormen Stressbedingungen heranwachsen zu müssen. Wir wollten, neben der Entschärfung eines aktuellen problematischen Beziehungskonfliktes, die positive Besetzung des kleinen Kindes unterstützen, um weiteren Entwicklungsstörungen bei allen drei Kindern vorzubeugen.

Den Eltern wurde ein Raum geboten, in dem sie ihre ambivalenten Gefühle ungestraft ausbreiten konnten. So werden sie vor Kurzschluss-Handlungen bewahrt und erleben eine therapeutische Atmosphäre, die ihnen bei zukünftigen Problemen einen Einstieg in weitere therapeutische Bearbeitung von Konflikten erleichtert.

Mit einem zweiten kürzeren Fallbeispiel möchte ich eine fast klassische Konstellation beschreiben, die häufiger in Eltern-Kleinkindtherapien anzutreffen ist. Es geht darum, Eltern ihre Konflikte mit dem Kind bewusst zu machen und sie von Blockierungen in ihrer Elternschaftsentwicklung zu befreien.

\section{$4 \quad$ Zweite Fallgeschichte}

Eine Kollegin wurde durch unsere Öffentlichkeitsarbeit auf meine Elternschaftstherapie bei der Stiftung Mütterhilfe aufmerksam und berichtete mir von einer Patientin, die Grossmutter sei und sich Sorgen um die Beziehung 
zwischen ihrer Tochter und dem kleinen 20 Monate alten Enkelsohn Manuel mache.

Die junge Mutter, 25 Jahre alt, nahm Kontakt mit mir auf und erzählte mir am Telefon, dass beide Eltern manchmal Angst vor ihrem so unbeherrschten kleinen Sohn hätten. Er greife sie richtiggehend an und schlage drauflos. Ich lud beide Eltern für den übernächsten Tag ein, mit ihrem Sohn zu kommen. Die Kindsmutter meinte aber, dass dieser Zeitpunkt ihrem Mann wohl nicht passe. Als ich genauer verstehen wollte, warum Freitagabend nicht gehe, hörte ich von der Mutter, dass ihr Mann nur sehr schlecht Deutsch spreche und sowieso gegenüber psychologischer Hilfe skeptisch eingestellt sei. Sie wollte mich erst mal allein kennenlernen und mir den Jungen zeigen. Diese Erfahrung mache ich oft, dass die Mütter den Therapeuten zunächst einmal alleine «beschnuppern» wollen. Ich verstehe es so, dass auf der einen Seite die Mutter überprüfen will, ob ihre mütterliche und weibliche Wahrnehmung vom männlichen Therapeuten genügend verstanden wird und dass sie zudem noch spüren will, ob dieser Mann in der Lage ist, ihren Partner erfolgreich emotional «abzuholen».

Die Mutter kam verspätet in hektischer Stimmung zum abgemachten Termin. Sie hatte die Praxis nicht gleich gefunden und sich noch telefonische Lotsenhilfe bei mir holen müssen.

Der kleine Manuel versteckte sich am Anfang im Wartezimmer verschüchtert hinter seiner Mama. Ich sprach ihn direkt an, erklärte ihm, dass seine Mama mit mir telefoniert und mir erzählt habe, dass sie sich über ihr häufiges Streiten Sorgen mache. Ich bat beide ins Therapiezimmer. Manuel hatte den kleinen Stuhl im Wartezimmer entdeckt und packte ihn. Die Mama wollte es ihm erst verbieten, ich aber erklärte, dass es doch eine gute Idee von Manuel sei, einen für ihn passenden Stuhl mitzunehmen.

Oft sind die uns aufsuchenden Eltern schon längere Zeit so gestresst, dass sie die kreativen Seiten im Verhalten ihrer Kinder gar nicht mehr anerkennen können.

Ich vernahm dann von einer sehr erschöpften Mutter, dass es seit 3 Wochen immer wieder ganz heftige Trotzanfälle gebe und der kleine Junge total unzufrieden wirke. Er habe Papa und Mama geschlagen und in letzter Zeit hätten sie auch schon mal zurückgehauen. Das hätten beide Eltern nie gewollt, aber nun wüssten sie nicht mehr weiter, erzählte die Mama verschämt. Ich würdigte ihren Mut, offen darüber zu sprechen und ihre Entschlossenheit, sich Hilfe zu holen. Ich sagte zu Manuel, dass ich gehört habe, Mama wolle ihn nicht hauen und dass wir jetzt darüber reden würden, warum das passiere. Er dürfe sich gern etwas zum Spielen 
aussuchen. Ich zeigte ihm das Regal, in dem für ihn geeignetes Spielmaterial zu finden war. Er nahm sich ein paar Tierfiguren, spielte mit diesen und liess die Mama mit mir sprechen.

Mama: «Es macht mir Angst, dass er so viel Wut in sich hat!»

Ich überlegte, was wohl vorgefallen sein könnte, dass das wütende Verhalten eines Kleinkindes von den Eltern so bedrohlich erlebt wird. Ich dachte, dass die Mama womöglich durch das Verhalten von Manuel auf besondere Weise gereizt werde, weil sie etwas in seinem Verhalten wahrnehme, das sie an etwas Anderes, sehr Traumatisierendes erinnere.

Ich lag mit meinerVermutung richtig. Mein Nachfragen hatte ergeben, dass die junge Mutter mit Schrecken realisiert hatte, wie ähnlich Manuel ihrem um ein Jahr älteren Bruder sei. Sie berichtete von einer anstrengenden Familiengeschichte, aus der ich das Wichtigste kurz zusammenfasse: Der ältere Bruder der Mutter war das grosse Sorgenkind der Familie. Woher dessen Probleme ursprünglich stammen, weiss Frau S. nicht, aber sie erinnert sich, dass er total verwöhnt worden sei und die Eltern ständig seine Fehler zu überspielen versucht und ihm alle Steine aus dem Weg geräumt hätten. Mit der Lehrstellensuche habe es auch nicht geklappt, doch man habe die Probleme nie beim Bruder gesucht, stattdessen hätten die Eltern ihn vor jeglicher Kritik in Schutz genommen. Heute sei er quasi arbeitsunfähig. Der Vater beschäftige ihn in seinem eigenen handwerklichen Betrieb und lasse viele Fehler des Sohnes durchgehen.

Ich staunte unverhohlen über die wichtige Entdeckung, dass in Manuels schwierigem Verhalten wahrscheinlich zu schnell der Bruder der Mutter gesehen wurde. Die Mama fing an zu begreifen, öffnete sich noch mehr und erzählte, wie sie sich schon einmal bei einem Streit vor Verzweiflung ins Badezimmer geflüchtet und die Tür verschlossen habe, worauf Manuel wütend geweint und vor der Tür erbrochen habe.

Wenn der Konflikt zwischen Eltern und Kind nicht geklärt wird, kann solch ein Verhalten zu ernsten vegetativen Störungen mit Krampfanfällen und heftigem Erbrechen führen. Daher ist eine rechtzeitig einsetzende, aufklärende therapeutische Intervention dringend notwendig.

Der kleine Manuel zeigte glücklicherweise in unserer ersten Sitzung eine seiner typischen Schwierigkeiten. Er wandte sich mit einem Spielzeug, mit dem er nicht zurechtkam, an die Mama. Er war sichtlich unzufrieden und forderte auf Italienisch etwas von ihr, was ich nicht verstehen konnte. Die Mutter: «Sehen Sie, so ist er dann, wenn etwas nicht geht!» Ich liess mir das Problem in Ruhe erklären und verstand folgendes: Der kleine Bub hatte eine spannende Idee, nämlich wollte 
er einem Playmobil-Motorradfahrer einen Beifahrer auf den Hintersitz montieren, was ihm aber nicht gelang. Ich bestätigte dem kleinen Manuel die gute Idee, und versuchte ihm zu helfen, aber leider gelang es auch mir nicht. Nun mussten wir beide die Unmöglichkeit des Unterfangens einsehen, was Manuel mit meiner Hilfe gut ertragen konnte.

Ich sagte zur Mama, dass wir damit etwas ganzWichtiges miteinander erlebt hätten. Es gehe darum, Manuel zu helfen, gut spielen zu können und Grenzen zu ertragen. Und es sei ganz wichtig, dass er in ihrem Erleben wirklich der Manuel sei und in ihrer Wahrnehmung nicht zu ihrem Bruder Antonio werde. Das war das Wesentliche an diesem ersten Kontakt. Zum weiteren Verlauf der Therapie erschienen beide Eltern. DerVater war sehr dankbar über das zunehmendeVerständnis der Zusammenhänge, und die junge Familie begann, sich besser von den Erwartungen der Herkunftsfamilie der Mutter und den möglichen Projektionen auf ihr Kind abzugrenzen. Eine reifere, konstruktivere Elternschaft liess sich aufbauen. Beide Eltern waren stolz auf ihren, bei mir im Therapieraum kreativ spielenden Sohn.

Nach zwei Sitzungen mit der ganzen Familie, zwei Mutter-KindTherapiestunden und einer längeren telefonischen Beratung der Mutter für einen geeigneten Umgang mit ihrem psychiatrisch auffälligen Bruder, den ich schliesslich erfolgreich zu einem erfahrenen Kollegen überweisen konnte, liess sich meine fokussierte Arbeit abschliessen.

Bei der Arbeit mit dieser Familie wurde ein wichtiger Ansatz der Pionierin für Eltern-Baby-Therapien Selma Fraiberg bestätigt. Sie arbeitete in den 50er Jahren in Chicago und kreierte den Begriff vom «Gespenst im Kinderzimmer». Gemeint sind gefährliche, eine gesunde Entwicklung behindernde Projektionen der Eltern aufs Kind. Auch auf Manuel lag solch eine belastende Zuschreibung. In ihm wurde der so schwierige und bedrohliche Bruder der Mutter gesehen. Wir schafften es gemeinsam, dieses «Gespenst» $\mathrm{zu}$ bannen.

\section{$5 \quad$ Zusammenfassung und Schlussbemerkung}

Aus meiner stationären und ambulanten psychotherapeutischen Arbeit heraus ist in Zusammenarbeit mit einigen psychoanalytischen KollegInnen schon in den 80er Jahren, parallel zur Kinderpsychotherapie, ein starkes Interesse an psychoanalytischer Elternarbeit entstanden. Aus dieser Auseinandersetzung mit der Notwendigkeit psychoanalytischer Elternarbeit, zeitgleich zur Einzelbehandlung der Kinder, hat sich bei mir das starke Interesse an der Arbeit mit dem frühen Sozialisationsfeld entwickelt. In diesem so prägenden und sensiblen Entwicklungsfeld reizt mich auch die kultur- und gesellschaftspolitische Dimension 
(vgl. Elias 1987). Werdende, junge Eltern erleben häufig mit ihren Kindern ein ungenügendes Holding in unserer Gesellschaft. Uns Psychoanalytikern aber stünde ein breites Wahrnehmungsrepertoire zur Verfügung. Wir müssen triebtheoretische Aspekte, ich-psychologische Beobachtungen, bindungstheoretische Dimensionen, Wissen über die Affektregulierung, beziehungsanalytische und selbstpsychologische sowie familiendynamische und kulturanalytische Betrachtungsweisen in die Arbeit im frühen Beziehungsfeld einbeziehen.

Aus diesen Betrachtungsweisen erwachsen auch Forderungen an die Gesellschaft.

Als mögliche Behinderungen einer gesunden Elternschaft und Generativität, möchte ich nur noch ein paar Stichworte liefern:

, Isolation im «Goldenen Käfig Kleinfamilie».

, Zunehmende Stressbelastung von Frauen und Männern in der Arbeitswelt mit parallel einhergehendem Verlust libidinöser Energie für Beziehungen in der Familie.

, Verunsicherung gut ausgebildeter Frauen hinsichtlich der Frage, wie sie Muttersein und berufliche Identität miteinander verbinden können.

, Inwieweit übernimmt der Staat mehrVerpflichtung für eine verantwortungsvolle, gute familienergänzende Betreuung von Babys und Kleinstkindern? Werden differenzierte entwicklungspsychologische Forschungsergebnisse ernst genommen und bei der Ausstattung von Sozialisationsräumen für Kinder als Grundlage herangezogen?

Nicht zuletzt bin ich wegen meiner gesellschafts- und kulturpolitischen Interessen als Psychoanalytiker in der Elternschaftstherapie tätig.

\section{Literatur}

Abelin, Ernst L. (1986): Die Theorie der frühkindlichen Triangulation. In: Jochen Stork, Hrsg., Das Vaterbild in Kontinuität und Wandlung, 45-72. Stuttgart: frommann-holzboog.

Bauriedl, Thea (1994): Auch ohne Couch. Psychoanalyse als Beziehungstheorie und ihre Anwendungen. Stuttgart: Verlag Internationale Psychoanalyse Cotta'sche Buchhandlung.

Dammasch, Frank (2008): Triangulierung und Geschlecht. Das Vaterbild in der Psychoanalyse und die Entwicklung des Jungen. In: Frank Dammasch, Dieter Katzenbach, Jessica Ruth, Hrsg., Triangulierung. Lernen, Denken und Handeln aus psychoanalytischer und pädagogischer Sicht, 13-39. Frankfurt: Brandes\&Apsel. 
Dolto, Francoise (1987): Das unbewusste Bild des Körpers. Berlin: Quadriga.

Downing, George (1996): Körper und Wort in der Psychotherapie. München: Kösel.

Elias, Norbert (1987): Die Gesellschaft der Individuen. Frankfurt: Suhrkamp Verlag.

Fonagy, Peter, György Gergely, Elliot L. Jurist, Mary Target (2004): Affektregulierung, Mentalisierung und die Entwicklung des Selbst. Stuttgart: Klett-Cotta.

Garstick, Egon (2001):Vom Elternwerden zur Elternschaft. Erster Erfahrungsbericht aus dem Projekt Elternschaftstherapie. In: Fernanda Pedrina, Hrsg., Beziehung und Entwicklung in der frühen Kindheit. Psychoanalytische Interventionen in interdisziplinären Kontexten, 31-51. Tübingen: edition diskord.

King, Vera (2004): Die Entstehung des Neuen in der Adoleszenz. Wiesbaden:VSVerlag für Sozialwissenschaften.

Knott, Maria. (2003): Statistisches Material aus der Babyambulanz Stuttgart. In: Analytische Kinder- und Jugendlichenpsychotherapie, 4/2003, 527-544. Frankfurt: Brandes \& Apsel.

Olivier, Christiane (1988): Jokastes Kinder. Die Psyche der Frau im Schatten der Mutter. Düsseldorf: Claassen.

Rodulfo, Ricardo (1996): Kinder-gibt es die? Die lange Geburt des Subjektes. Freiburg i. Brsg.: Kore.

Spitz, René A. (1976): Vom Dialog: Studien über den Ursprung der menschlichen Kommunikation und ihrer Rolle in der Persönlichkeitsbildung. Stuttgart: Ernst Klett.

Stork, Jochen (1986): Der Vater - Störenfried oder Befreier? In: Jochen Stork, Hrsg., Das Vaterbild in Kontinuität und Wandlung, 9-30. Stuttgart: frommannholzboog.

Winnicott, Donald (1974): Reifungsprozesse und fördernde Umwelt. München: Kindler. 